Ophthalmologe 2011 · 108:805-806

DOI 10.1007/s00347-011-2329-6

Online publiziert: 21. August 2011

(c) Springer-Verlag 2011

\author{
T. Reinhard \\ Augenklinik, Universitätsklinikum Freiburg
}

\section{Perspektiven der Hornhauttransplantation}

\author{
Neue Operations- und \\ Transplantationstechniken
}

In der Hornhauttransplantation konnten in den vergangenen Jahren vielversprechende Operationstechniken eingeführt werden. Zwar ist das klare Hornhauttransplantatüberleben bei konventioneller perforierender Keratoplastik, beispielsweise mit geführten Trepanationssystemen, ausgezeichnet. In Standardsituationen bleiben über 10 Jahre postoperativ deutlich mehr als 90\% der Transplantate klar [1]. Auch ist die Zufriedenheit der Patienten mit dem erreichten Ergebnis sehr gut [2]. Eine kritische Beleuchtung vermag jedoch auch Schattenseiten der bisherigen Operationstechniken festzustellen.

Dies betrifft beispielsweise Fadenkomplikationen, die am besten vermeidbar sind, wenn keine Fäden mehr nötig wären oder diese sehr frühzeitig entfernt werden könnten [3]. Vor allem betrifft dies jedoch die optische Versorgung nach Entfernung aller Fäden. So muss ein durchschnittlicher postoperativer keratometrischer Astigmatismus von 4-6 dpt ausgeglichen werden [2]. Der größte Teil der konventionell transplantierten $\mathrm{Pa}$ tienten benötigt also postoperativ zumindest eine Brille. Viele Patienten wären mit einer (formstabilen) Kontaktlinse besser versorgt, kommen mit dieser jedoch manchmal nicht gut zurecht.

Alle Bestrebungen bei Standardtransplantationen gehen derzeit dahin, die Rehabilitationsphase zu verkürzen sowie sie so einfach wie möglich zu gestalten. Dies umfasst Modifikationen der perforierenden ebenso wie die Einfüh- rung lamellierender Transplantationstechniken.

Mit dem Femtosekundenlaser können mikrometergenau nicht nur gerade, sondern v. a. profilierte Schnitte ausgeführt werden. Beliebt sind das Tophat- sowie das Zigzag-Profil. Die profilierten Schnitte ermöglichen postoperativ größere Wundflächen und damit ein schnelleres Einheilen der Transplantate. Dies wiederum ist mit der Möglichkeit der deutlich früheren Fadenentfernung verbunden. Somit kann mithilfe des Femtosekundenlasers eine deutliche Verbesserung des derzeitigen Status der konventionellen perforierenden Keratoplastik erreicht werden. Eine Reduzierung des keratometerischen Astigmatismus nach Fadenentfernung ist bislang jedoch nicht nachweisbar (siehe Birnbaum in diesem Heft). Grund hierfür ist aller Wahrscheinlichkeit nach die Applanation der Kornea durch das Interface des Lasers. Dies führt möglicherweise zu unterschiedlichen Konfigurationen im Spender- und Empfängerbereich.

Genau dieses Problem lässt sich bei Verwendung des Excimerlasers vermeiden. Bei diesem handelt es sich um das derzeit einzige "Schneidesystem“, das Kontakt mit dem Auge vermeidet und völlig berührungsfrei arbeitet. Zwar lassen sich so keine profilierten Schnitte erzeugen. Die Hornhautfäden können also erst recht spät, ähnlich wie bei der konventionellen perforierenden Keratoplastik, entfernt werden. Es ist jedoch ein deutlicher Vorteil im Hinblick auf den resultierenden postoperativen irregulären und re- gulären keratometrischen Astigmatismus nach Fadenentfernung feststellbar (siehe Seitz in diesem Heft).

Bei endothelialen Erkrankungen der Augenhornhaut, beispielsweise bei der Fuchs-Endotheldystrophie oder bei der bullösen Degeneration können hintere lamelläre Verfahren eingesetzt werden. Dies betrifft zum einen die DSAEK („Descement stripping automated endothelial keratoplasty“), bei der meist 8-9 mm im Durchmesser große Scheibchen mit einer Dicke von 100-150 $\mu \mathrm{m}$ nach Entfernen der kranken inneren Hornhautschichten, also Endothel und Descemet, mit Luft an die vorderen Hornhautschichten des Empfängers nahtfrei „angedrückt“ werden. Bei der DMEK („Descement membrane endothelial keratoplasty") ist das Spendermaterial mit etwa $15 \mu \mathrm{m}$ noch dünner. Die Vorteile dieser Methoden liegen auf der Hand: Die Architektur der Empfängerhornhaut bleibt im Wesentlichen erhalten. Im Rahmen der in Kleinschnitttechnik durchgeführten Einbringung des Transplantates ist nicht mit wesentlichen postoperativen regulären oder irregulären Astigmatismen zu rechnen. Da zudem keine Fadenentfernung erforderlich ist, ist die Rehabilitation der Patienten im Vergleich zu den perforierenden Verfahren sehr kurz. Nachteilig ist, dass bei der DSAEK im Bereich des Aufeinandertreffens von Wirts- und Spenderhornhaut Trübungen und Lichtbrechungsunregelmäßigkeiten auftreten können. Dies beeinflusst die erreichbare Sehschärfe manchmal deutlich. Bei DSAEK 
und DMEK werden zudem im stromalen Bereich bereits vorliegende sekundäre Veränderungen, also Vernarbung bei länger bestehenden Ödemen nicht entfernt. Auch dies kann durchaus mit Visuslimitierungen einhergehen. Schließlich ist im Hinblick auf die bislang kurzen Nachbeobachtungszeiten noch unklar, wie sich der in der frühen postoperativen Phase im Vergleich zur konventionellen perforierenden Transplantation erhöhte Endothelzellverlust langfristig auswirken wird. Ohne Frage hat die hintere lamelläre Hornhauttransplantation noch ein erhebliches Entwicklungspotenzial (siehe Heinzelmann in diesem Heft).

Bei Hornhauterkrankungen, die nur das Stroma, nicht jedoch Endothel und Descemet betreffen, kann im Rahmen einer tiefen anterioren Keratoplastik DALK („deep anterior lamellar keratoplasty“) der kranke Teil der Augenhornhaut ersetzt werden. Dies hat den Vorteil, dass das eigene Endothel verbleibt und nicht für Abstoßungen - die allerdings bei den infrage kommenden Indikationen, wie beispielsweise dem Keratokonus, eher selten sind - Angriffsziel sein kann. Entscheidend für die DALK ist, dass die Wirtsdescemet komplett von stromalen Anteilen befreit wird. Geschieht dies nicht, sind Trübungen und Lichtbrechungsunregelmäßigkeiten im Bereich des Aufeinandertreffens von Wirts- und Spenderhornhaut mit entsprechenden Visuslimitierungen der meist jungen Patienten die Regel (siehe Cursiefen in diesem Heft).

Neben der Entwicklung neuer Operationstechniken in Standardsituationen hat es in den vergangenen Jahren auch deutliche Fortschritte im Bereich der Hochrisikotransplantation insbesondere bei Patienten mit ausgeprägten Oberflächenstörungen, beispielsweise bei Limbusstammzellinsuffizienz, gegeben. Die autologe Transplantation hat bei einseitigen Erkrankungen ebenso wie die allogene Transplantation adulter Limbusstammzellen bei beidseitiger Erkrankung in den vergangenen Jahren an manchen Kliniken standardisiert Einzug in die klinische Versorgung gehalten. Postoperativ ist bei allogener Vorgehensweise - und bei der deutlichen Mehrzahl der Patienten handelt es sich um eine beidseitige Erkrankung - mit einem hohen Abstoßungsrisiko zu rechnen. Dies macht die Verwendung gematchter Transplantate sowie einer postoperativen intensiven lokalen und systemischen Immunsuppression erforderlich. Die Verwendung autologer Zellen, beispielsweise aus der Mundschleimhaut, und eine anschließende Transdifferenzierung zu Limbusstammzellen der Kornea sind daher ein wichtiges Ziel der Forschung. Auf dem Weg dahin ist es ganz entscheidend, möglichst viel über die Molekularbiologie von Limbusstammzellen zu erfahren (siehe Eberwein in diesem Heft).

Die vielfältigen und fantastischen neuen chirurgischen Möglichkeiten eröffnen für unsere Patienten mit Hornhauterkrankungen neue Perspektiven. Bei allem Enthusiasmus darf jedoch nicht vergessen werden, dass sich der Großteil dieser Entwicklungen erst langfristig in der klinischen Versorgung bewähren muss. Ihr

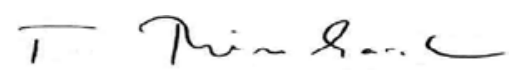

Prof. Dr. T. Reinhard

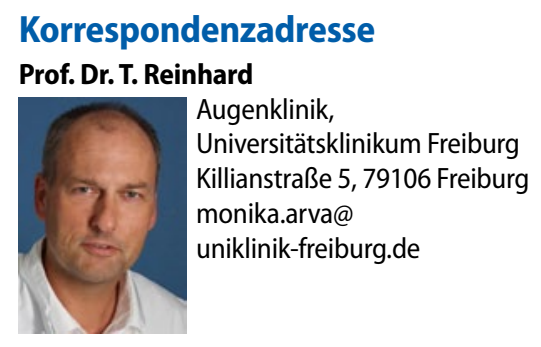

\section{Literatur}

1. Reinhard T, Böhringer $D$, Hüschen $D$, Sundmacher R (2002) Chronischer Endothelzellverlust des Transplantats nach perforierender Keratoplastik: Einfluss der Migration von Endothelzellen vom Transplantat zur Wirtshornhaut. Klin Monatsbl Augenheilkd 219:410-416

2. Böhringer D, Schindler A, Reinhard T (2006) Funktion und Zufriedenheit nach perforierender Keratoplastik - Ergebnisse eine Fragebogenzensus. Ophthalmologe 103:677-681

3. Böhringer D, Sundmacher R, Reinhard T (2010) Fadenkomplikationen nach perforierender Keratoplastik. Klin Monatsbl Augenheilkd 227:735-738

\section{Auslöser für Lebersche Kongeni- tale Amaurose entdeckt}

Kinder, die von der seltenen, unheilbaren Netzhauterkrankung Lebersche Kongenitale Amaurose (LCA5) betroffenen sind, erblinden oft schon während des ersten Lebensjahrs aufgrund genetischer Defekte in den Fotorezeptorzellen. Einem internationalen Forschungsteam des Universitätsklinikums Tübingen ist es gelungen, den Erkrankungsmechanismus von LCA5 in molekularen und zellphysiologischen Prozessen im Auge zu entschlüsseln.

Es sind mindestens 15 verschiedene Gene bekannt, deren Mutation zu LCA5 führen können. Da trotz dieser Heterogenität ein eng umrissenes Krankheitsbild entsteht, geht man von einem gemeinsamen, überlappenden Ursprung aus. Eine zentrale Rolle spielt hierbei der intraflagellare Transport (IFT). Über diesen gelangen Proteine, die für den Sehvorgang gebraucht werden, von ihrer Produktionsstelle, dem inneren Segment des Fotorezeptors, in das lichtempfindliche äußere Segment. Der Transport geschieht hierbei durch Motorproteine über ziliare Mikrotubuli-Bahnen. Die Forscher untersuchten zwei bekanntermaßen mit LCA5 in Verbindung stehende Mutationen in Lebercilin - einem bis dahin wenig charakterisierten Protein, das sich hauptsächlich im Bereich des Ciliums nachweisen lässt.

Sie stellten fest, dass die Bindung von Lebercilin an die Motorprotein-Komplexe für den Transportvorgang in Sehzellen notwendig ist. Ein Vergleich des normalen Proteins mit den zwei mutierten Proteinvarianten zeigte, dass die Bindungsfähigkeit der defekten Proteine an beide Komplexe durch die Mutation verloren geht. Als Konsequenz der herabgesetzten Bindungsfähigkeit an die IFT-Maschinerie wird das Transportsystem nicht beladen. Der Transport von Sehpigment und anderer essentieller Proteine, die für Ausbildung und Funktionsfähigkeit des äußeren Segments notwendig sind, unterbleibt und führt zum Absterben der Fotorezeptorzellen. Da eine Veränderung des Lebercilin-Proteins jedoch nicht zu Transportstörungen an Zilien im gesamten Organismus führt, konnte die Vermutung bestätigt werden, dass Lebercilin kein struktureller Bestandteil des IFT-Systems ist. Mit diesen Ergebnissen hat eine beteiligte Gruppe aus den USA unmittelbar damit begonnen, eine Gentherapie für LCA5 zu entwickeln.

Literatur: Boldt K, Mans DA, Won J et al (2011) Disruption of intraflagellar protein transport in photoreceptor cilia causes Leber congenital amaurosis in humans and mice. J Clin Invest $121: 2169-2180$ 